\title{
Underwater Endoscopic Mucosal Resection without Submucosal Injection Facilitates En bloc Resection of Colon Adenomas Extending into a Diverticulum 亩
}

\author{
Yoshikazu Hayashi', Masahiro Okada, ${ }^{1,2}$, Takaaki Morikawa', Tatsuma Nomura', Hisashi Fukuda', Takahito Takezawa', Alan \\ Kawarai Lefor ${ }^{3}$ and Hironori Yamamoto ${ }^{1}$ \\ ${ }^{1}$ Division of Gastroenterology, Department of Medicine, Jichi Medical University, Shimotsuke, ${ }^{2}$ Department of Gastroenterology, Shin- \\ Oyama City Hospital, Oyama, ${ }^{3}$ Department of Surgery, Jichi Medical University, Shimotsuke, Japan
}

Superficial colonic neoplasms sometimes extend into a diverticulum. Conventional endoscopic mucosal resection of these lesions is considered challenging because colonic diverticula do not have a muscularis propria and are deeply inverted. Even if the solution is carefully injected below the mucosa at the bottom of the diverticulum, the mucosa is rarely elevated from the diverticular orifice, and it is usually just narrowed. Although endoscopic submucosal dissection or full-thickness resection with an over-the-scope clip device enables the complete resection of these lesions, it is still challenging, time consuming and expensive. Underwater endoscopic mucosal resection without submucosal injection (UEMR) is an innovative technique enabling en bloc resection of superficial colon lesions. We report three patients with colon adenomas extending into a diverticulum treated with successful UEMR. UEMR enabled rapid and safe en bloc resection of colon lesions extending into a diverticulum. Clin Endosc 2021;54:436-440

Key Words: Colonic diverticulum; Colonic neoplasm; Underwater endoscopic mucosal resection

\section{INTRODUCTION}

Colorectal cancer was the third most common cancer and the second most common cause of cancer death worldwide in 2018. ${ }^{1}$ A national polyp study revealed that colonoscopic removal of colorectal adenomas can reduce the incidence and mortality of colorectal cancer., ${ }^{2,3}$ Since then, the role of endoscopic resection of colorectal adenomas has been greatly enhanced. However, superficial colorectal neoplasms sometimes occur where endoscopic removal is technically difficult. A lesion extending inside a colonic diverticulum is a difficult

Received: June 20, 2020 Revised: July 25, 2020

Accepted: August 3, 2020

Correspondence: Hironori Yamamoto

Division of Gastroenterology, Department of Medicine, Jichi Medical University, 3311-1 Yakushiji, Shimotsuke, Tochigi 329-0498, Japan

Tel: +81-285-58-7347, Fax: +81-285-40-6598, E-mail: ireef@jichi.ac.jp

ORCID: https://orcid.org/0000-0002-3601-1153

(c) This is an Open Access article distributed under the terms of the Creative Commons Attribution Non-Commercial License (http://creativecommons.org/ licenses/by-nc/3.0) which permits unrestricted non-commercial use, distribution, and reproduction in any medium, provided the original work is properly cited. scenario because of the lack of the muscularis propria. Some of these patients undergo surgical resection ${ }^{4}$ or advanced endoscopic treatment such as endoscopic submucosal dissection (ESD). Binmoeller et al. first reported underwater endoscopic mucosal resection without submucosal injection (UEMR), an innovative technique enabling en bloc resection of superficial colon lesions. ${ }^{5,6}$ The first case of UEMR for a colon adenoma extending into a diverticulum was reported in $2020{ }^{7}$ We report successful UEMR of colorectal adenomas extending into a diverticulum.

\section{CASE REPORTS}

Patient 1 was a 65 -year-old man with a positive fecal immunochemical test who underwent colonoscopy showing a flat lesion extending into a diverticulum in the cecum. The lesion was $1.5 \mathrm{~cm}$ in diameter and suspicious for an adenoma. After water immersion, the mucosa in the diverticulum was everted through the orifice and the diverticulum-side demarcation of the lesion was easily identified. We snared the entire lesion and 
safely resected it in an en bloc fashion (Fig. 1, Supplementary Video 1).

Patient 2 was a 70 -year-old woman with a positive fecal immunochemical test who underwent colonoscopy revealing a flat lesion extending into a diverticulum in the ascending colon. The lesion was $1.5 \mathrm{~cm}$ in diameter and suspicious for an adenoma. After water immersion, part of the lesion was everted from the diverticulum and further everted using the edge of a distal cap. We snared the entire lesion and safely resected it in an en bloc fashion (Fig. 2, Supplementary Video 1).
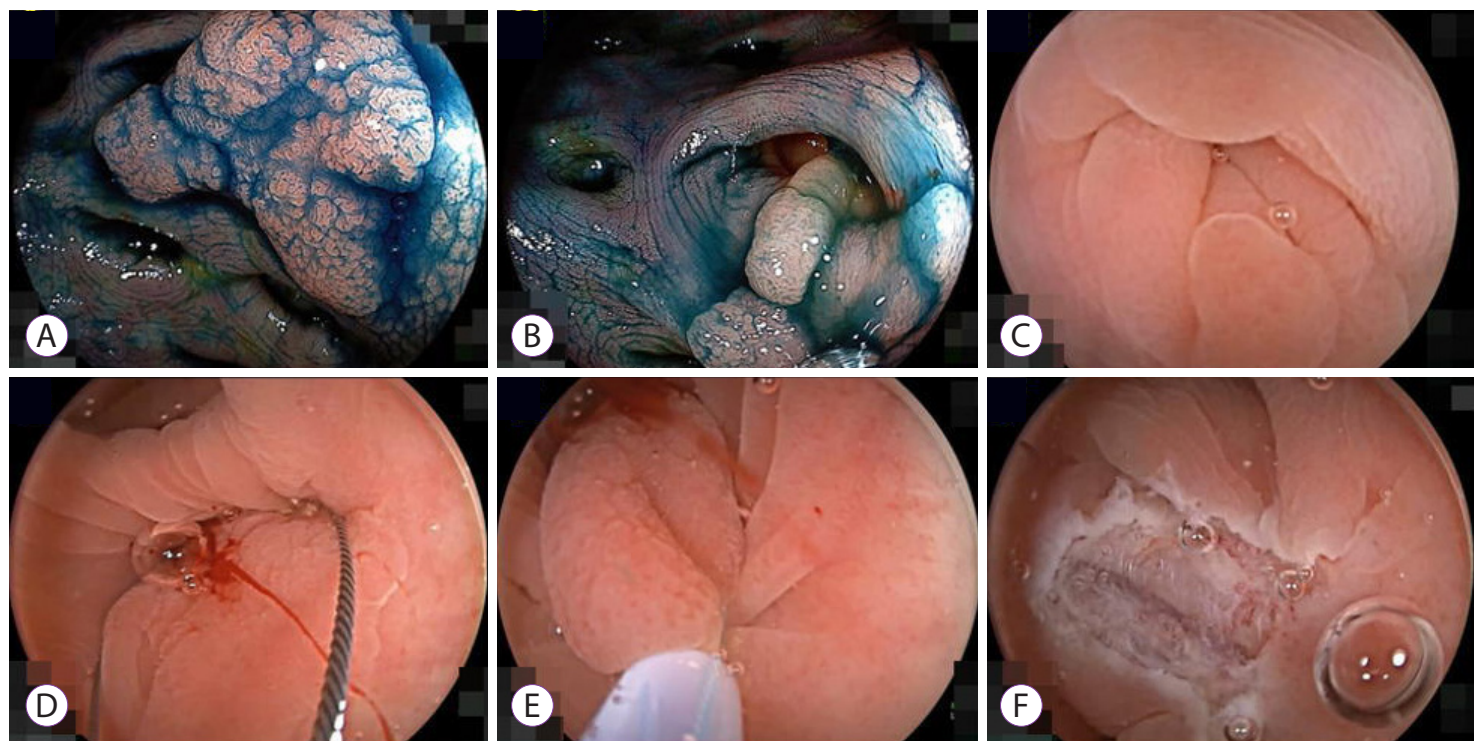

Fig. 1. Underwater endoscopic mucosal resection without submucosal injection of a lesion in the cecum. (A) A 1.5-cm flat lesion in the cecum. (B) This lesion extended into a diverticulum. (C) The border of the lesion in the diverticulum was identified using water immersion. (D) The snare tip was anchored outside the lesion in the diverticulum. (E) The entire lesion was snared and cut using pure-cut mode diathermy. $(F)$ The mucosal defect without any residual lesion was closed with hemoclips. Histology of the resected specimen showed an adenoma with negative resection margins.
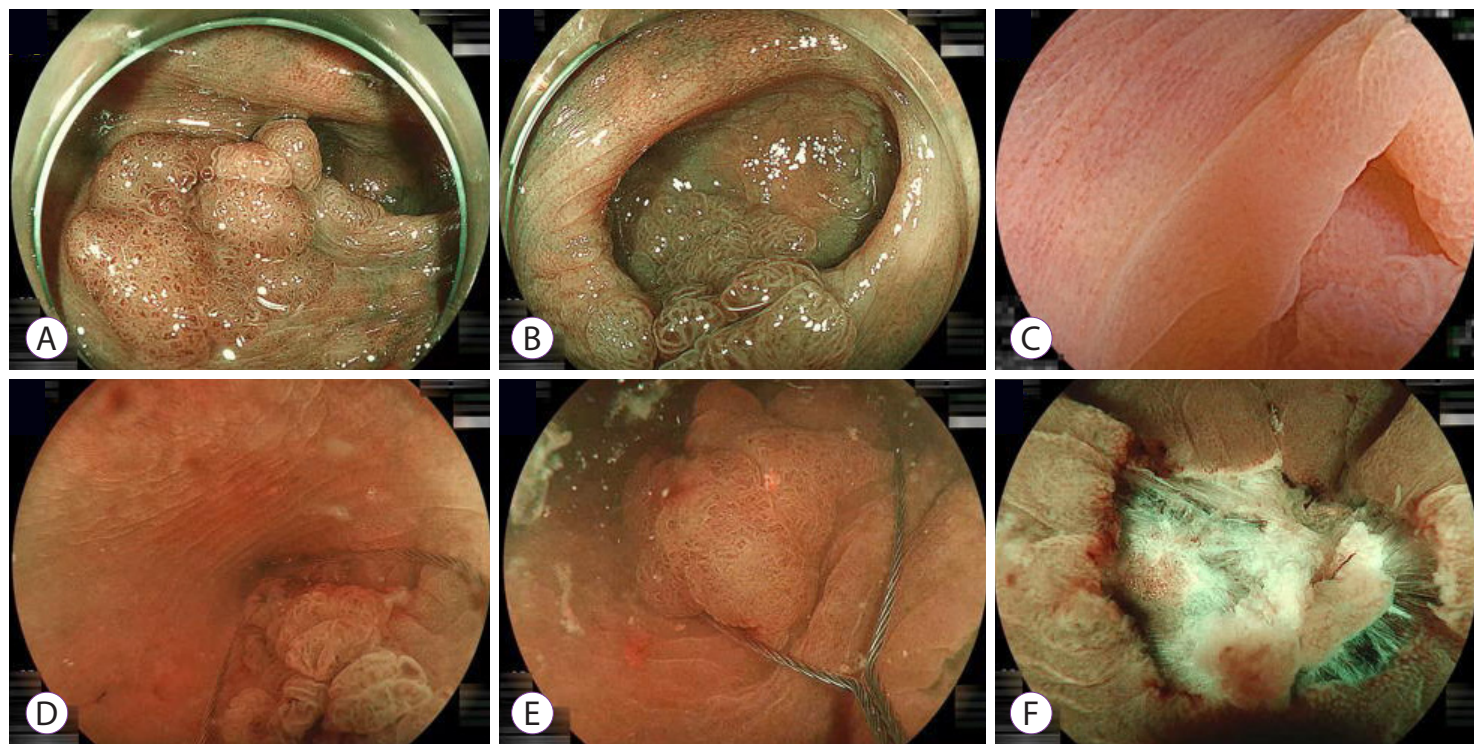

Fig. 2. Underwater endoscopic mucosal resection without submucosal injection of the lesion in the ascending colon. (A) A 1.5-cm flat lesion in the ascending colon. (B) This lesion extended into a diverticulum. (C) Lesion demarcation in the diverticulum was done after everting the lesion with edge of a distal cap after water immersion. (D) The snare tip was placed outside the lesion in the diverticulum. (E) The entire lesion was snared and cut using pure-cut mode diathermy. (F) The mucosal defect without any residual lesion was closed using a reopenable clip with an 11-mm opening width (SureClip; Micro-Tech, Nanjing, China). Histology of the resected specimen showed an adenoma with negative resection margins. 




Fig. 3. Underwater endoscopic mucosal resection without submucosal injection of the lesion in the ascending colon. (A) A 2-cm flat lesion in the ascending colon. (B) This lesion extended into a diverticulum. (C) Lesion demarcation in the diverticulum was done after water immersion. (D) The snare tip was placed outside the lesion in the diverticulum. The entire lesion was snared and cut using pure-cut mode diathermy. (E) The mucosal defect without any residual lesion. (F) The defect was completely closed with hemoclips while maintaining water immersion. Histology of the resected specimen showed an adenoma with negative resection margins.

Patient 3 was an 80-year-old man who underwent routine colonoscopy before ESD for gastric cancer, which revealed a flat lesion extending into a diverticulum in the ascending colon. The lesion was $2 \mathrm{~cm}$ in diameter and suspicious for an adenoma. After water immersion, the mucosa in the diverticulum was everted through the orifice and the diverticulum-side demarcation of the lesion was easily identified. We snared the entire lesion and safely resected it in an en bloc fashion (Fig. 3, Supplementary Video 1).

All procedures were performed on an outpatient basis. Endoscopic treatment utilized a distal attachment (Olympus, Tokyo, Japan), 15-mm RotaSnare (Medi-Globe GmbH, Achenmühle, Germany), distilled-water irrigation and pure cutting mode diathermy. All post-UEMR mucosal defects were closed with hemoclips immediately while maintaining water immersion. There were no adverse events in any patient. Pathologic evaluation of all lesions showed adenomas with negative margins. The three cases in this report are all of the consecutive patients in our experience of UEMR for colonic lesions extending into a diverticulum. Therefore, we have successfully achieved en bloc resection in all cases without failure so far.

\section{DISCUSSION}

Superficial colonic neoplasms sometimes extend into a diverticulum. Conventional endoscopic mucosal resection (EMR) of these lesions is considered challenging because colonic diverticula lack a muscularis propria and are deeply inverted. Even if the solution is carefully injected below the mucosa at the bottom of the diverticulum during EMR, the mucosa is rarely elevated from the diverticular orifice and is usually just narrowed. It is very difficult to resect neoplasms extending into a diverticulum in an en bloc fashion using conventional EMR.

UEMR is an innovative technique enabling en bloc resection of superficial colon lesions. The colonic mucosa and submucosa appear to float above the circular muscularis propria in the underwater view of endoscopic ultrasound. Therefore, even a flat lesion can be easily snared. The essential point to perform UEMR is to completely aspirate the intestinal gas in order to collapse the intestinal lumen and subsequently fill it with water like when performing endoscopic ultrasound.

UEMR has some advantages compared to conventional EMR; it allows one to reliably snare the entire lesion in a reasonable time without concern for dispersion of the injected solution leading to flattening of the mucosal elevation, as 
seen in the EMR procedure. Dispersion of the injected solution makes snaring a flat lesion more difficult or impossible. ${ }^{5}$ UEMR also allows one to snare a lesion repeatedly until the entire lesion is captured, sometimes with contraction. ${ }^{8}$ The EMR procedure sequence includes two irreversible steps: elevating the mucosa with an injection and snaring of the elevated mucosa. Each step demands a certain level of skill. If one of the steps fails, it will be difficult to achieve EMR with a complete en bloc resection of the lesion.

We recommend cutting the captured lesion with pure-cut mode diathermy to avoid thermal injury to the muscularis propria. When cutting a flat lesion with pure-cut mode, there is no need to worry about immediate massive bleeding because these lesions rarely have feeding arteries which would massively bleed. Meanwhile, too much thermal injury in a diverticulum presents a risk of post-polypectomy coagulation syndrome because of the lack of the muscularis propria. After removing the lesion, the postprocedural mucosal defect can be closed with fewer hemoclips because the defect stays smaller with water immersion than with gas insufflation.

If massive bleeding occurs immediately, blood will quickly interfere with endoscopic visibility. We recommend the use of a water-irrigating pump to easily control the water supply and clear the endoscopic view. We also recommend the preparation of hemoclips before cutting, to deal with any immediate bleeding. If possible, a reopenable clip is ideal.

There has been a report of perforation associated with $\mathrm{UEMR}^{9}$ probably caused by stretching of the colonic wall with a retroflexed colonoscope. When the lesion was snared after submucosal injection even after water immersion, differing from UEMR, the colonic wall stretched and the procedure resulted in a perforation. ${ }^{10}$ In our opinion, accidental intramuscular injection may also allow snaring of the muscularis propria of the lesion even with water immersion. Although ESD or endoscopic full-thickness resection with an over-the-scope clip device ${ }^{11,12}$ enables complete resection of superficial colonic lesions extending into a diverticulum, it is still challenging, time consuming and expensive. Some recently introduced traction devices facilitate ESD of the lesions. ${ }^{13-15}$

This report is the first case series of UEMR of colon adenomas extending into a diverticulum. UEMR enabled rapid and safe en bloc resection of colon adenomas extending into a diverticulum without adverse events. UEMR may be useful for endoscopic removal of colonic neoplasms extending into a diverticulum

\section{Conflicts of Interest}

Hironori Yamamoto was supported by honoraria, grants, and royalties from Fujifilm Corporation and has a consultant relationship with the company.
Funding

None.

Author Contributions

Conceptualization: Yoshikazu Hayashi

Methodology: YH, Masahiro Okada, Takaaki Morikawa, Tatsuma Nomura, Hisashi Fukuda, Takahito Takezawa

Project administration: $\mathrm{YH}$

Supervision: Alan Kawarai Lefor, Hironori Yamamoto

Visualization: $\mathrm{YH}$

Writing-original draft: $\mathrm{YH}$

Writing-review\&editing: $\mathrm{YH}, \mathrm{AKL}, \mathrm{HY}$

ORCID
Yoshikazu Hayashi:

Masahiro Okada:

Takaaki Morikawa:

Tatsuma Nomura:

Hisashi Fukuda:

Takahito Takezawa:

Alan Kawarai Lefor:

Hironori Yamamoto:
https://orcid.org/0000-0003-2393-639X https://orcid.org/0000-0001-8766-0762 https://orcid.org/0000-0002-9051-0519 https://orcid.org/0000-0003-1243-8846 https://orcid.org/0000-0002-0294-4676 https://orcid.org/0000-0002-8181-3307 https://orcid.org/0000-0001-6673-5630 https://orcid.org/0000-0002-3601-1153

Supplementary Material

Video 1. Underwater endoscopic mucosal resection without submucosal injection of the colon lesions (https://doi.org/10.5946/ce.2020.168.v001).

\section{REFERENCES}

1. Ferlay J, Soerjomataram I, Ervik M, et al. GLOBOCAN 2012 v1.0, cancer incidence and mortality worldwide: IARC CancerBase No. 11 [Internet]. Lyon: International Agency for Research on Cancer; c2013 [updated 2017 Aug 23]. Available from: https://publications.iarc.fr/ Databases/Iarc-Cancerbases/GLOBOCAN-2012-Estimated-CancerIncidence-Mortality-And-Prevalence-Worldwide-In-2012-V1.0-2012

2. Winawer SJ, Zauber AG, Ho MN, et al. Prevention of colorectal cancer by colonoscopic polypectomy. The National Polyp Study Workgroup. N Engl J Med 1993;329:1977-1981.

3. Zauber AG, Winawer SJ, O’Brien MJ, et al. Colonoscopic polypectomy and long-term prevention of colorectal-cancer deaths. N Engl J Med 2012;366:687-696.

4. Imai $\mathrm{K}$, Hotta $\mathrm{K}$, Ono $\mathrm{H}$. Unusual colonic mucosal cancer extending into a diverticulum. Dig Endosc 2014;26:752.

5. Binmoeller KF, Weilert F, Shah J, Bhat Y, Kane S. "Underwater" EMR without submucosal injection for large sessile colorectal polyps (with video). Gastrointest Endosc 2012;75:1086-1091.

6. Yamashina T, Uedo N, Akasaka T, et al. Comparison of underwater vs conventional endoscopic mucosal resection of intermediate-size colorectal polyps. Gastroenterology 2019;157:451-461.e2.

7. Shiotsuki K, Imai K, Hotta K, Ito S, Kishida Y, Ono H. Underwater endoscopic mucosal resection for complete R0 removal of an adenoma extending deep into a colonic diverticulum. Endoscopy 2020;52:E374-E375.

8. Lee RF, Nomura T, Hayashi Y, Okada M, Yamamoto H. En bloc removal of a colonic polyp using progressive polyp contraction with underwater endoscopic mucosal resection: the PP-CUE technique. Endoscopy 2020;52:E434-E436.

9. Ponugoti PL, Rex DK. Perforation during underwater EMR. Gastrointest Endosc 2016;84:543-544.

10. Kawamura T, Sakai H, Ogawa T, et al. Feasibility of underwater endo- 
scopic mucosal resection for colorectal lesions: a single center study in Japan. Gastroenterology Res 2018;11:274-279.

11. Jimenez-Garcia VA, Yamada M, Ikematsu H, et al. Endoscopic submucosal dissection in management of colorectal tumors near or involving a diverticulum: a retrospective case series. Endosc Int Open 2019; 7:E664-E671.

12. Shakhatreh MH, Hair C, Shaib YH, Green LK. Removal of a colonic polyp in a diverticulum: a novel use of the over-the-scope clip device. Gastrointest Endosc 2015;81:756.

13. Shichijo S, Yamasaki Y, Takeuchi Y. Case of colonic adenoma involving a diverticulum resected by a traction-assisted endoscopic submucosal dissection technique. Dig Endosc 2017;29:729-730.

14. Kato M, Uraoka T, Wada M, et al. Laterally spreading tumor involving a colon diverticulum successfully resected by endoscopic submucosal dissection. Gastrointest Endosc 2016;84:191-192.

15. Albouys J, Geyl S, Charissoux A, et al. Counter-traction using clips and rubber banding for endoscopic submucosal dissection of a laterally spreading tumor involving a diverticulum in the colon. Endoscopy 2019;51:E295-E296. 